LA-UR-00-6115

Approved for public release; distribution is unlimited.

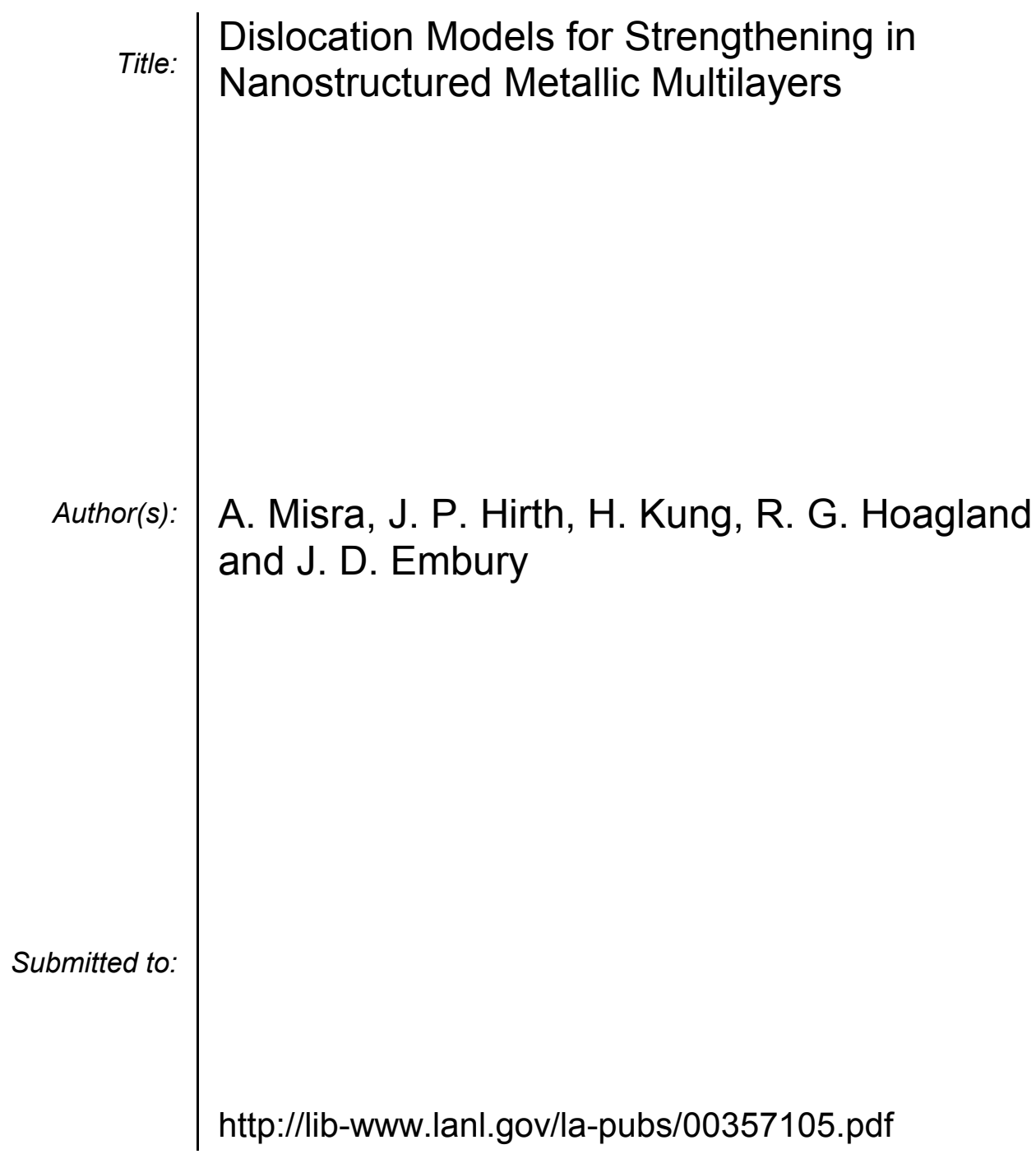

Los Alamos National Laboratory, an affirmative action/equal opportunity employer, is operated by the University of California for the U.S. Department of Energy under contract W-7405-ENG-36. By acceptance of this article, the publisher recognizes that the U.S. Government retains a nonexclusive, royaltyfree license to publish or reproduce the published form of this contribution, or to allow others to do so, for U.S. Government purposes. Los Alamos National Laboratory requests that the publisher identify this article as work performed under the auspices of the U.S. Department of Energy. Los Alamos National Laboratory strongly supports academic freedom and a researcher's right to publish; as an institution, however, the Laboratory does not endorse the viewpoint of a publication or guarantee its technical correctness. 


\title{
Dislocation Models for Strengthening in Nanostructured Metallic Multilayers
}

\author{
A. Misra, J. P. Hirth, H. Kung, R. G. Hoagland and J. D. Embury, \\ MST Division, Los Alamos National Laboratory, MS K765, Los Alamos, NM 87545
}

\begin{abstract}
Ultra-high strength metallic multilayers are ideal for investigating the effects of length scales in plastic deformation of metallic materials. Experiments on model systems show that the strengths of these materials increase with decreasing bilayer period following the Hall-Petch model. However, as the layer thickness is reduced to the nm-scale, the number of dislocations in the pile-up approaches one and the pile-up based Hall-Petch model ceases to apply. For nm-scale semi-coherent multilayers, we hypothesize that plastic flow occurs by the motion of single dislocation loops, initially in the softer layer, that deposit misfit type dislocation arrays at the interface and transfer load to the harder phase. The stress concentration eventually leads to slip in the harder phase, overcoming the resistance from the misfit arrays at the interface. A model is developed within the framework of classical dislocation theory to estimate the strengthening from this mechanism. The model predictions are compared with experimentally measured strengths.
\end{abstract}

\section{INTRODUCTION}

Metal-metal composites, synthesized by co-deformation, electroplating or vapor deposition, possess strengths that approach $1 / 2$ to $1 / 3$ of the theoretical limit when the microstructural length scales are on the order of a few nanometers [1,2]. In some cases, the maximum strength of these composites may be an order of magnitude higher than the strength of the soft constituent phases. A fundamental understanding of the deformation mechanisms at nm-length scales is needed to allow optimum microstructural design of these nanostructured materials for desired applications, such as structural components in microelectromechanical systems (MEMS).

For single-phase metals, the increase in yield strength $(\sigma)$ with decreasing grain size $(d)$ is interpreted by means of the Hall-Petch (H-P) model based on dislocation pile-ups:

$\sigma=\sigma_{0}+\mathrm{kd}^{-1 / 2}$

where $\sigma_{0}$ represents the lattice friction stress and $\mathrm{k}$ (H-P slope) indicates the relative hardening contribution from grain boundaries. This model is also applicable if hardness data is used instead of $\sigma$. For lamellar composites, the increase in yield strength with decreasing interphase boundary spacing (h) is also described by the H-P model for the case where $h$ replaces $d$ in eq. (1). Several recent experimental [1,3] and theoretical [4-6] studies have, however, shown that the H-P model may break down at nm-length scales. At $\mu \mathrm{m}$-length scales, dislocation pile-ups can be treated as a continuum distribution and eq. (1) holds. At the nm-length scales, pile-ups are discrete and the exponent in eq. (1) departs from 0.5 . The assumption that the number of dislocations in the pileup $(\mathrm{N})$ decreases in proportion with decreasing $\mathrm{h}$ provides one way to interpret the dependence of strength on $\mathrm{h}$ at nm-length scales [4,5]. In this approach, a peak in strength is reached when $\mathrm{h}$ is so small that $\mathrm{N}$ cannot exceed one $[4,5]$. This approach assumes that the dislocation sources are such that pile-ups will always form, whether $\mathrm{N}=2$ or significantly greater.

An alternate view is that continuum pile-ups form at large $\mathrm{h}$ and, at nm-length scales, deformation behavior is governed by single dislocations [7]. Single dislocations may propagate 
in the softer phase by an Orowan bowing process, similar to yielding in thin films on substrates [8], and transfer load to the harder phase till it yields or fractures [7].

In this investigation, we extend the Embury-Hirth [7] model for single dislocation behavior in fine-scale composites to nm-scale semi-coherent metallic multilayers. The roles of the misfit dislocations at the interface and the in-plane coherency stresses in determining the Orowan stress are calculated. Also, calculated is the interface resistance to single dislocation transmission. The model predictions are compared with experimental results on $\mathrm{Cu}-\mathrm{Ni}$ systems.

\section{RESULTS AND DISCUSSION}

\section{Hall-Petch Behavior}

We first present experimental results on a number of $\mathrm{Cu}$-based multilayers to determine the length scale limits to which H-P model is valid. These multilayers were all synthesized by physical vapor deposition techniques (sputtering or evaporation), mechanical properties were evaluated by nanoindentation and microstructures were characterized by transmission electron microscopy (TEM). The details of the experimental procedures are described elsewhere [3]. Fig. 1 shows data for $\mathrm{Cu}-\mathrm{Nb}, \mathrm{Cu}-\mathrm{Cr}$ and $\mathrm{Cu}-\mathrm{Ni}$ systems as log-log plots of $\left(\mathrm{H}-\mathrm{H}_{0}\right)$ vs. h. Here $\mathrm{H}$ is the hardness of the multilayer, $\mathrm{H}_{0}$ is the hardness of a $1 \mu \mathrm{m}$ thick $\mathrm{Cu}$ film, and $\mathrm{h}$ is the layer thickness (or, one-half of the bilayer period). It follows from eq. (1) that a linear fit with a slope of -0.5 is consistent with H-P model. For all three systems, H increases with decreasing $\mathrm{h}$ according to eq. (1) but deviations from the H-P behavior are observed at nm-length scales. Two observations are made from these data. First, in Fig. 1, the y-intercept of the linear fit gives k, the H-P slope. $\mathrm{k}$ defines the rate of hardening with decreasing h, i.e., the effectiveness of the boundaries as obstacles to slip transmission. While k correlates with shear modulus for the $\mathrm{Cu}-\mathrm{Cr}$ and $\mathrm{Cu}-\mathrm{Nb}$ systems, $\mathrm{k}$ for $\mathrm{Cu}-\mathrm{Ni}$ and $\mathrm{Cu}-\mathrm{Nb}$ are about the same, even though $\mu_{\mathrm{Ni}}>\mu_{\mathrm{Nb}}$. This indicates that other factors such as dislocation structure of the interface, yield strength of the harder phase and the misorientation of the slip systems could also

be important in determining k. For example, a cube-on-cube orientation relation in the fcc $\mathrm{Cu}$-fcc Ni single crystalline system results in no misorientation between slip systems, and this special orientation boundary would be a relatively weaker obstacle. Second, the H-P break down length scale decreases as the lattice parameter mismatch at the interface increases. $\mathrm{Cu}-\mathrm{Nb}$ has the highest mismatch of $\sim 10 \%$ between the $\{110\} \mathrm{Nb}$ and $\{111\}$ $\mathrm{Cu}$ planes that make the interface plane, and exhibits $\mathrm{H}-\mathrm{P}$ down to $\sim 10 \mathrm{~nm}$. $\mathrm{Cu}-\mathrm{Cr}$ and $\mathrm{Cu}-\mathrm{Ni}$ have mismatch of $\sim 2.5 \%$ and exhibit $\mathrm{H}-\mathrm{P}$ breakdown at $\mathrm{h} \approx 40-50 \mathrm{~nm}$. Furthermore, the $\mu$ mismatch between layers appears to have no significant effect on the value of $\mathrm{h}$ at H-P breakdown.

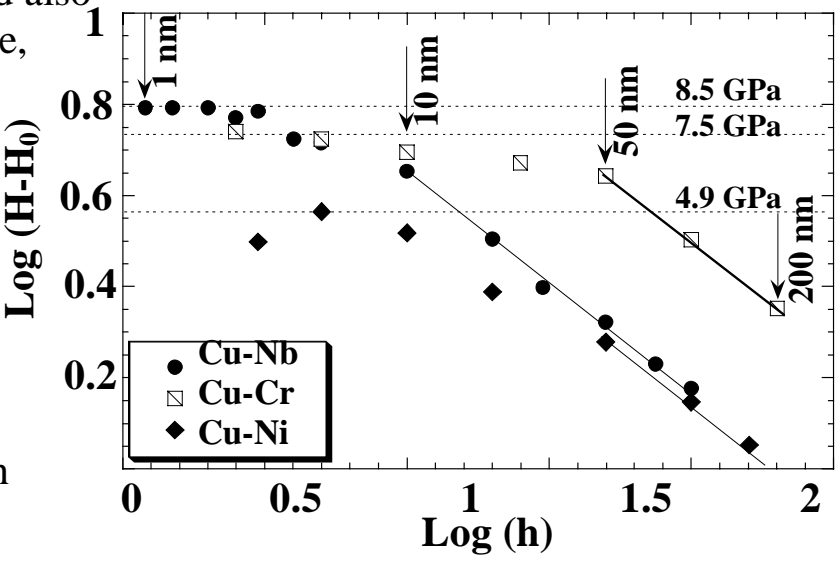

Fig. 1 Plot of $\log \left(\mathrm{H}-\mathrm{H}_{0}\right)$ vs. $\log \mathrm{h}$ for $\mathrm{Cu}$-based multilayers, where $\mathrm{H}$ is the multilayer hardness, $\mathrm{H}_{0}$ is $\mathrm{Cu}$ film hardness and $\mathrm{h}$ is one-half of bilayer period. Dotted horizontal lines indicate the maximum $\mathrm{H}$ of each of the 3 systems. Linear fits have slope of -0.5 indicating that $\mathrm{H}$ increases with decreasing $h$ according to Hall-Petch law, but this model is not applicable at $\mathrm{h}<\sim 10-50 \mathrm{~nm}$. 
These results are consistent with our earlier theoretical work on estimation of H-P breakdown length scales in polycrystalline multilayers [6]. After the H-P model breaks down, in all systems, the hardness continues to increase to a maximum as $\mathrm{h}$ is decreased from a few tens of $\mathrm{nm}$ to a few nm. A model for strengthening at nm-scales is described next.

\section{Single Dislocation Model}

The model developed here applies to multilayers deformed under iso-strain conditions, with one phase significantly harder than the other and the length scale such that dislocation pileups do not form. Plasticity initiates in the softer phase when its yield strength is reached (Fig. 2(a)), while the harder phase continues to deform elastically. In this condition, the stress-strain curve deviates from linearity but strain hardening is high as plasticity is confined to only one phase and load is transferred to the harder phase. Yielding in the harder phase occurs at a higher applied stress when dislocations from softer phase can cross over the interface (Fig. 2(b)). In the limited tensile tests conducted on nm-scale multilayers, the stress-strain curves did not reveal a well-defined single yield point, consistent with our hypothesis [9]. Furthermore, multilayers deformed to small plastic strains reveal dislocations predominantly in the softer phase suggesting that the harder phase has not yielded. An example of this behavior is shown Fig. 3 (TEM micrograph) that correlates with the schematic of Fig. 2(a). This free-standing $\mathrm{Cu}-\mathrm{Nb}$ multilayer was annealed at $600{ }^{\circ} \mathrm{C}$ for 1 hour and the thermal stress (calculated strain is $\sim 0.5 \%$ ) induced during cooling has caused yielding of the softer $\mathrm{Cu}$ layer where an array of dislocation loops are observed. No significant dislocation activity is detected in the Nb layer at this strain level.

We first describe the calculation of the Orowan stress for the motion of the hairpin dislocation in layer A of thickness $\mathrm{h}$. This stress is given as follows:

$$
\tau_{\text {Orowan }}=\frac{2 \mathrm{~W}_{\mathrm{D}}}{\mathrm{bh}}
$$

where $W_{D}$ is the energy per unit length of the dislocation, and $b$ the Burgers vector. To calculate $\mathrm{W}_{\mathrm{D}}$, the approach is as follows. Consider the hairpin dislocation loop shown in Fig. 4(a). We assume that it is a $60^{\circ}$ dislocation on a $\{111\}$ glide plane and the interface plane is $\{100\}$.
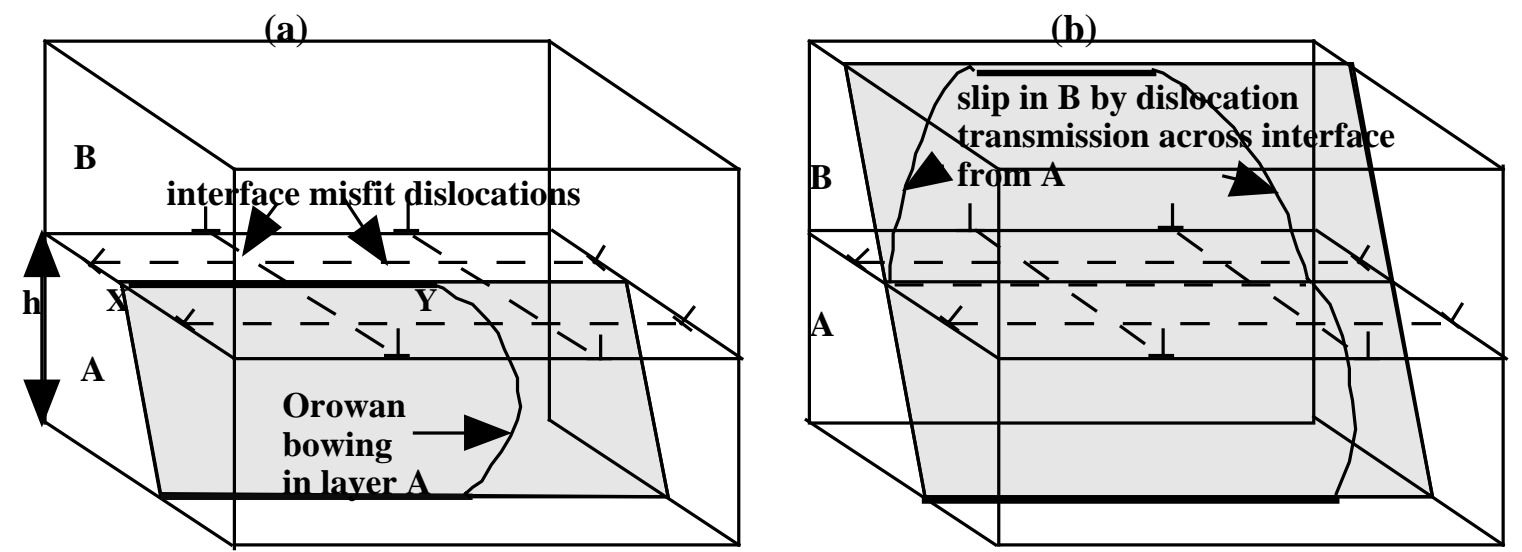

Fig. 2 Schematic illustration of the deformation mechanism in nm-scale multilayers; (a) Orowan bowing of a glide dislocation loop in the softer layer (A) while the harder phase deforms elastically (glide plane is shown shaded). The motion of the glide dislocation leaves behind misfit-type dislocations (e.g., the segment labeled XY) at the interface that already has a cross-grid of misfit dislocations. (b) At higher applied stress, composite yields plastically when single dislocations can transmit across the interfaces. 
The bowed segment in Fig. 4(a) is near-screw and the segment deposited at the interface is edge. For this loop, the view with the glide plane edge-on is shown in Fig. 4(b). One can mathematically treat the two edge dislocations deposited at the interface by the motion of the glide loop by creating infinitesimal dislocation dipoles and separating them to the desired positions [10]. If the plastic strain in the layer $\mathrm{A}$ is $\varepsilon^{\mathrm{A}}$ prior to yielding in $\mathrm{B}$, then there will be an array of these glide loops of average spacing $\left(b / \varepsilon^{A}\right)$. One finds the value of $\mathrm{W}_{\mathrm{D}}$ for this array by integrating the Peach-Koehler force $(=\sigma b)$ on the dislocations over the layer thickness:

$\mathrm{W}_{\mathrm{D}}=-\int_{0}^{h} \sigma \mathrm{b} \mathrm{dy}$

The dislocation line energy expressions for simple stretch or shear arrays have been derived by Kreidler

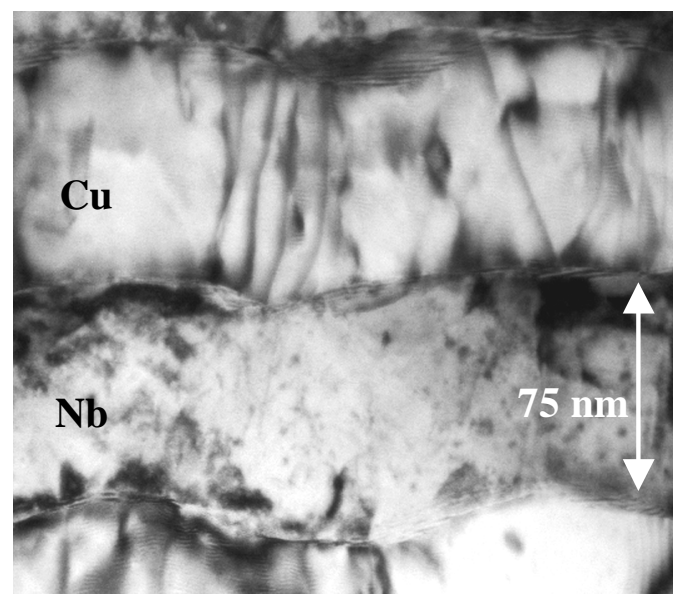

Fig. 3 TEM micrograph of a $\mathrm{Cu}-\mathrm{Nb}$ multilayer annealed at $600{ }^{\circ} \mathrm{C}$. Thermal strains $(\sim 0.5 \%)$ result in an array of dislocation loops in the $\mathrm{Cu}$ layer, while no significant plasticity is observed in $\mathrm{Nb}$. and Anderson [11]. Here we have considered $60^{\circ}$ glide dislocations and carried out the integration separately for the three resolved components of $b\left(b_{y}\right.$ normal to the interface and $b_{x}$ and $b_{z}$ along the line directions of the cross-grid of misfit dislocations). For each component, the integration is the sum of integrals over paths I and II shown in Fig. 4(b). We further define an upper bound and a lower bound estimate for $\mathrm{W}_{\mathrm{D}}$. In the upper bound estimate, the work done against the stress fields of both arrays of the misfit dislocations, and the self-energy of the glide loops are included. In the lower bound estimate, we assume that for the $h$ range where this model applies, the misfit dislocation spacing $(\lambda)$ is greater than the equilibrium value. TEM observations of the $\mathrm{Cu}-\mathrm{Ni}$ interface have shown that even when $\mathrm{h}$ is an order of magnitude higher than the critical thickness $\left(h_{c}\right), \lambda$ is a factor of 2 above the equilibrium value of $\sim 10 \mathrm{~nm}$ [12]. Thus, the glide of the hairpin loops adds the "missing" misfit dislocations at the interface and hence "negative" work is being done as $\lambda$ is reduced to its equilibrium value. This negative work effectively cancels out the calculated work against the stress field of equilibrium misfit arrays, and to a first approximation, we need only consider the self-energy terms in the lower bound estimate. The result is a sum of the self-energies of the dislocation components $b_{x}, b_{y}$ and $b_{z}$ :

$\mathrm{W}_{\mathrm{D}}=\frac{\mu \mathrm{b}^{2}}{8 \pi(1-v)} \ln \frac{\left(\mathrm{h}^{2}+\mathrm{L}^{2}\right)^{1 / 2}}{\mathrm{~b}}+\frac{\mu \mathrm{b}^{2}}{4 \pi(1-v)} \ln \frac{\left(\mathrm{h}^{2}+\mathrm{L}^{2}\right)^{1 / 2}}{\mathrm{~b}}+\frac{\mu \mathrm{b}^{2}}{8 \pi} \ln \frac{\left(\mathrm{h}^{2}+\mathrm{L}^{2}\right)^{1 / 2}}{\mathrm{~b}}$

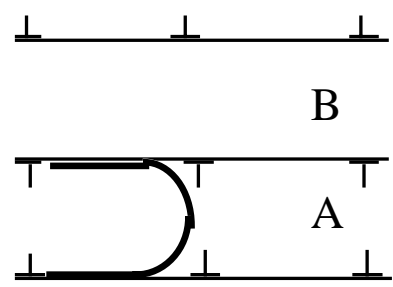

(a)

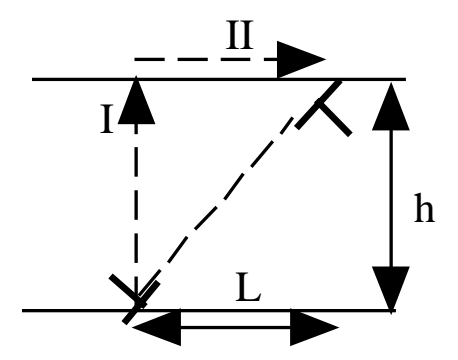

(b)
Fig. 4(a) Schematic of a glide dislocation moving by an Orowan bowing process in the softer layer A while the harder layer B continues to deform elastically; (b) view of the glide loop shown in (a) with glide plane edge-on. The deposition of two edge dislocations at the interface is equivalent, mathematically, to the creation of infinitesimal dislocation dipoles, followed by separation to the desired positions, along sequential paths I and II. 
This result, when substituted in eq. (2), should give a lower bound of the applied shear stress to move the hairpin dislocations in the softer layer, in the absence of any residual stresses. The details of the upper bound calculation and comparison with the lower bound will be presented in a separate article. To account for the effect of residual stress, we consider only the coherency stress here and ignore the intrinsic stress (growth-related stresses in PVD). The maximum biaxial coherency stress can be estimated from misfit strain by means of Hooke's law [10]. For $h>$ $h_{c}$, the coherency stress will decrease by a factor of $\left(h / h_{c}\right)[13]$. We assume that the presence of in-plane coherency stresses $\left(\tau_{\text {coh }}\right)$ will lower the barrier for the motion of hairpin dislocations in the softer layer. The effective applied stress to initiate slip in layer A is:

$$
\tau_{\text {applied }}^{\mathrm{A}}=\tau_{\text {Orowan }}-\tau_{\text {coh }}
$$

Once the array of hairpin loops has caused yielding in the softer phase, the resistance of the interface to single dislocation crossing needs to be computed. The lattice parameter mismatch at the interface $\left(\varepsilon_{\mathrm{m}}\right)$ when added to the plastic strain accumulated in the softer phase in the elasticplastic region $\left(\varepsilon^{\mathrm{A}}\right)$, gives the new misfit dislocation spacing as $\mathrm{b} /\left(\varepsilon_{\mathrm{m}}+\varepsilon^{\mathrm{A}}\right)$. The stress tensor for this array of edge dislocations is computed from equations by Hirth and Lothe [14], and rotated to the glide system co-ordinates. These stresses vary with distance parallel as well as normal to the edge array. We have calculated the stress tensor at a point that is mid-way between two misfits and a distance of $3 \mathrm{~b}$ normal to the interface. Once the glide dislocation is pushed out of the interface by a small distance of $\sim 3 b$, the repulsion from the same-sign dislocations in the array will push it further away. There will be additional attractive and repulsive forces, respectively, from the edge arrays at distances $h$ below and above the array from which the glide dislocation is being pushed out. These forces are roughly equal in magnitude and cancel out. Therefore, the effective applied stress is just what is needed to push the glide dislocation out of the interface by a small distance of $\sim 3 \mathrm{~b}$. This calculated stress $\left(\tau_{\text {array }}\right)$ when added to the Orowan stress (eq. 5) gives the composite shear strength:

$$
\tau^{*}=\tau_{\text {array }}+\tau_{\text {applied }}^{\mathrm{A}}
$$

This shear stress is converted to normal stress by means of the Schmid factor for $\{111\}<110\rangle$ glide in $\langle 100\rangle$ oriented fcc multilayers, and compared to experimental data on $\mathrm{Cu}-\mathrm{Ni}$ in Fig. 5. The solid data points in Fig. 5 are hardness values divided by a factor of 3, and the open points are tensile data on electrodeposited materials. For $h>h_{c}$, the model provides a reasonable fit to the experimental data. At larger h (sub- $\mu \mathrm{m}$ to tens of $\mu \mathrm{m})$, the pile-up based H-P model will be more appropriate. The single dislocation model incorporates several unit processes involved in the deformation of nm-scale multilayers such as Orowan bowing, coherency stresses, interface misfit dislocations array resistance, etc. Although not discussed here, the dislocation image force (Koehler) effects can be added to eq. (6) as appropriate. Atomistic simulations have shown that the Koehler barrier can be significantly altered by coherency stresses [19]. For $h<h_{c}$, and in general for $\mathrm{h}<\sim 5 \mathrm{~nm}$, the Orowan bowing stresses are on the order of theoretical strength limit, and this model will not work. We speculate that a saturation in strength will be reached at these length scales corresponding to the transmission of dislocations across interfaces overcoming the coherency and Koehler-type barriers. A drop in strength, as observed in miscible Cu-Ni systems, may occur if the width of the intermixed interface is on the order of $h$. The deformation mechanisms at these length scales $(\mathrm{h}<\sim 5 \mathrm{~nm}$ ) may be better studied through atomistic simulations $[19,20]$. 


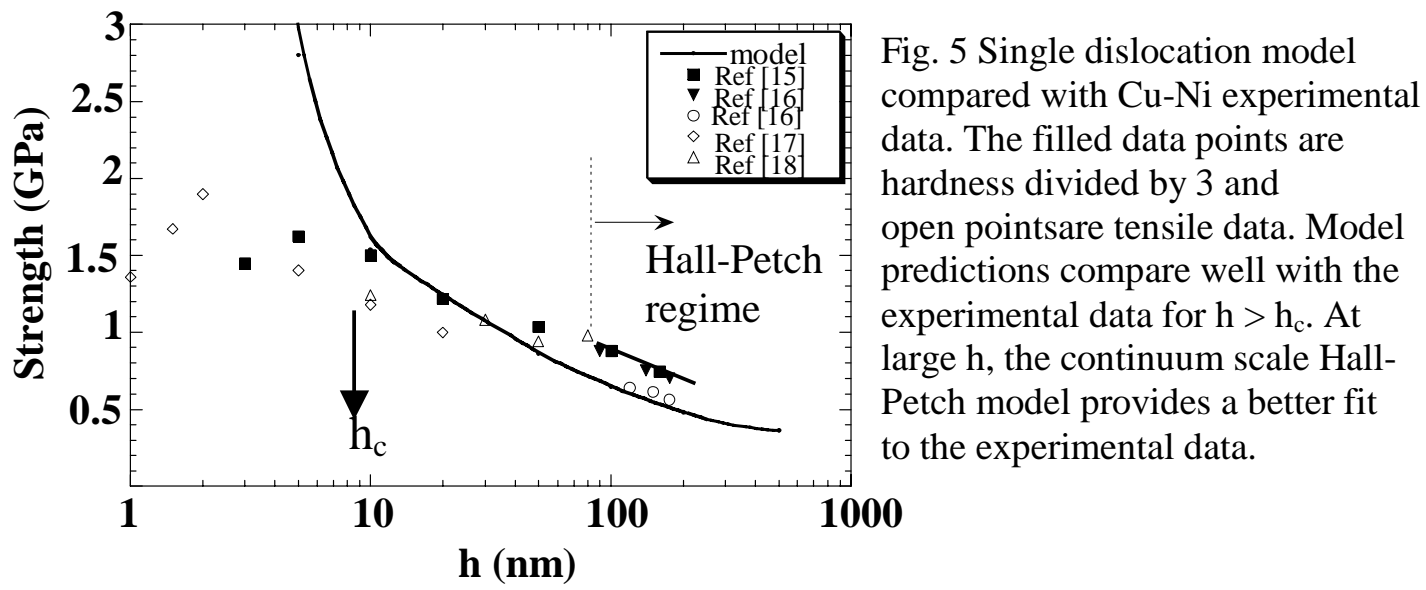

\section{SUMMARY}

The Hall-Petch model describes the strengthening in multilayers at near-micron length scales. At nm-scales, plasticity may involve motion of single, rather than piled-up, dislocations, and a model, based on Embury-Hirth [7], is developed to interpret this behavior. We calculate the applied stress for the motion of Orowan loops in the softer layer by incorporating effects of misfit dislocations at the interface and in-plane coherency stresses. This stress when added to the calculated interface resistance to single dislocation transmission gives the composite yield strength that compares favorably with experiments for semi-coherent multilayers.

This work was supported by the Office of Basic Energy Sciences, Department of Energy. Authors acknowledge discussions with M. Verdier, M. Nastasi and T.E. Mitchell.

\section{REFERENCES}

1. B.M. Clemens, H. Kung and S.A. Barnett, MRS Bulletin, 24, 20, February (1999).

2. P.M. Anderson, T. Foecke and P.M. Hazzledine, MRS Bulletin, 24, 27, February (1999).

3. A. Misra, M. Verdier, Y.C. Lu, H. Kung, T.E. Mitchell, M. Nastasi and J.D. Embury, Scripta Mat., 39, 555 (1998).

4. P.M. Anderson and C. Li, NanoStructured Materials, 5, 349 (1995).

5. S.I. Rao, P.M. Hazzledine and D. Dimiduk, Mat.Res.Soc.Sym.Proc., 362, 67 (1995).

6. A. Misra, M. Verdier, H. Kung, J.D. Embury and J.P. Hirth, Scripta Mat., 41, p 973 (1999).

7. J.D. Embury and J.P. Hirth, Acta Met., 42, 2051 (1994).

8. W.D. Nix, Scripta Mat., 39, 545 (1998).

9. H. Huang and F. Spaepen, Acta Mat., 48, 3261 (2000).

10. J.P. Hirth and X. Feng, J.Appl. Phys., 67, 3343 (1990).

11. E.R. Kreidler and P.M. Anderson, Mat.Res.Soc.Sym.Proc., 434, 159 (1996).

12. T. Yamamoto, LANL, unpublished research.

13. B. Shoykhet, M.A. Grinfeld and P.M. Hazzledine, Acta Mater., 46, 3761 (1998).

14. J.P. Hirth and J. Lothe, Theory of Dislocations, Krieger, p 734 (1992).

15. M. Verdier, M. Niewczas, J.D. Embury, M. Nastasi and H. Kung, Mat. Res. Soc. Sym. Proc., 522, 77 (1998).

16. R.F. Bunshah et al., Thin Solid Films, 72, 261 (1980).

17. S. Menezes and D.P. Anderson, J. Electrochem. Soc., 137, 440 (1990)

18. D.M. Tench and J.T. White, J. Electrochem. Soc., 138, 3757 (1991).

19. R.G. Hoagland, Phil.Mag.A, submitted.

20. S.I. Rao and P.M. Hazzledine, Phil. Mag.A, 30, 2011 (2000). 